\title{
Hyperbranched Polyglycerol Functionalized Silica Stationary Phase for Hydrophilic Interaction Liquid Chromatography
}

\author{
Hengye Li, ${ }^{*}$ Xuemeng Zhang, ${ }^{*}$ Lin Zhang, $* *$ Hui Cang, ${ }^{*}$ Fenying Kong, $*$ Dahe Fan, ${ }^{* \dagger}$ and \\ Wei WANG*广 \\ *School of Chemistry and Chemical Engineering, Yancheng Institute of Technology, No. 9 Yinbing Avenue, \\ Tinghu, Yancheng 224000, China \\ **Yancheng Entry-Exit Inspection and Quarantine Bureau, No. 85 Kaifang Avenue, Yancheng 224000, China
}

\begin{abstract}
Surface-initiated anionic-ring-opening multibranching polymerization was employed to prepare a hyperbranched polyglycerol (HPG) functionalized silica stationary phase for hydrophilic interaction liquid chromatography (HILIC). The obtained stationary phase was characterized by Fourier-transform infrared spectrometry (FT-IR) and thermogravimetric analysis (TGA). The chromatographic properties of the prepared stationary phase were systematically investigated. The abundance and multitude distribution of hydroxyl groups in HPG endowed the stationary phase with improved hydrophilicity and enhanced separation performance compared with the stationary phase functionalized with monolayer of hydroxyl groups. The stationary phase showed excellent retention of various polar compounds, such as nucleosides, necleobases, phenols and sulfanilamides, indicating great potential in the separation of complex biosamples.
\end{abstract}

Keywords Hyperbranched polyglycerol, surface-initiated anionic-ring-opening multibranching polymerization, stationary phase, hydrophilic interaction liquid chromatography

(Received September 21, 2017; Accepted November 15, 2017; Published April 10, 2018)

\section{Introduction}

Hydrophilic interaction liquid chromatography (HILIC) was firstly termed by Alpert in 1990 and it has been recognized as an alternative HPLC mode for the separation of polar compounds. ${ }^{1}$ Polar compounds are ubiquitous in complex biosamples, such as nucleosides in metabonomic samples, glycopeptides in proteinomic samples and saccharides in glycomics samples. HILIC is thus a promising alternative to traditional LC methods in bioanalysis. ${ }^{2,3}$

HILIC stationary phases have been developed rapidly with the growing popularity of HILIC., ${ }^{4,5}$ Silica gels modified with multitudinous polar functional groups, such as diol, ${ }^{6,7}$ amino, ${ }^{8}$ amide, ${ }^{9}$ zwitterionic moieties ${ }^{10,11}$ and saccharide, ${ }^{12}$ have been proposed as HILIC stationary phases for the separation of polar analytes $^{13}$ and inorganic anions. ${ }^{14}$ Monoliths based HILIC stationary phases have also been developed. ${ }^{15}$ However, most of these stationary phases are usually modified with monolayer of functional groups with relatively poor hydrophilicity, which limits their application to complex samples. ${ }^{16}$ Compared with monolayer stationary phases, stationary phases functionalized with hydrophilic multilayer polymer have attracted more attention owing to their high hydrophilicity and large binding capacity. ${ }^{17,18}$ As for hydrophilicity modifications of stationary phases with polymers, "grafting from" polymerization has been proved to be an efficient approach. It overmatches other polymerization methods concerning the generation of polymer

† To whom correspondence should be addressed.

E-mail: fandahe@ycit.cn; wangw@ycit.edu.cn chains with high grafting densities from initiators on the substrate. Various approaches have been employed for "grafting from" strategy, such as surface-initiated ionic polymerization, ${ }^{19}$ atom transfer radical polymerization (ATRP), ${ }^{20,21}$ reversibleaddition fragmentation chain transfer (RAFT) polymerization ${ }^{15}$ and click chemistry. ${ }^{22}$

Dendritic polymers (dendrimers and hyperbranched polymers), have highly branched structures and all bonds in dendritic polymers converge to a focal point or core. ${ }^{23}$ Theoretically, a branched polymer graft has higher hydrophilicity with the coated surface than that of its linear analogue at an equivalent grafting density. ${ }^{24}$ Compared with dendrimers, which usually require tedious multistep syntheses, ${ }^{25,26}$ hyperbranched polymers can be prepared in a one-pot synthesis, and can be a favorable alternative to dendrimers. ${ }^{27}$ Hyperbranched polymers show an advantage of an extremely high density of functional groups. Hyperbranched polymer functionalized stationary phases have been developed for HPLC applications in an affinity chromatography mode, ${ }^{28}$ a size exclusion chromatography mode $^{29}$ and ion-exchange chromatography. ${ }^{30,31}$ Hyperbranched polymer was also adopted to prepare a HILIC stationary phase. Peng et al. prepared a novel polyethylenimine (PEI) functionalized HILIC stationary phase through a "graft to" method. ${ }^{32}$ The column showed excellent separation performance for polar analytes under the HILIC mode due to the hyperbranched structure of PEI. Exploring hyperbranched polymers for novel HILIC stationary phases is intriguing and significant for the development and application of HILIC. Hyperbranched polyglycerols (HPG) are highly hydrophilic hyperbranched polymers, due to these compact and globular structures with multitude distribution of hydroxyl groups down 




$\mathrm{SiO}_{2} @ \mathrm{HPG}$

Fig. 1 Illustration of the preparation of the $\mathrm{SiO}_{2} @ \mathrm{HPG}$ stationary phase.

to the cavity levels. ${ }^{33}$ So, HILIC stationary phase functionalized with HPG would offer rich hydroxyl groups and a larger contact possibility with polar analytes relative to common hydroxyl stationary phases chemically bonded with small hydroxyl molecules. However, such an attempt has not yet been made.

In this work, we combined the merits of the "grafting from" strategy and HPG to prepare a HPG functionalized stationary phase for HILIC through surface-initiated anionic-ring-opening multibranching polymerization. The obtained stationary phase was well characterized, and its chromatographic characteristics under the HILIC mode was systematically investigated and compared with a control column with a monolayer of hydroxyl groups to assess the role of the HPG in the improved separation.

\section{Experimental}

\section{Reagents and materials}

Tris(hydroxymethyl)aminomethane (Tris), glycidol, potassium tert-butoxide (PTB), 3-chloropropyltrimethoxysilane (CPTMS), thymine, uracil, cytosine and guanine were purchased from J\&K Scientific Ltd. (Beijing, China). $\mathrm{SiO}_{2}$ (with particle size of $5 \mu \mathrm{m}$, pore size of $100 \AA$ and specific surface area of $290 \mathrm{~m}^{2} / \mathrm{g}$ ) was provided by Dalian Replete Scientific Instruments Co. Ltd. (Dalian, China). 2,5-Dimethylphenol (DMP), pyrocatechol (PC) and 3-nitrophenol (NP) were obtained from Energy Chemical (Shanghai, China). Sulfadimethoxine (SDM), sulfamethoxypyridazine (SMP), sulfafurazole (SIZ) and sulfamethizole (SMZ) were purchased from Dr. Ehrenstorfer $\mathrm{GmbH}$ (Augsburg, Germany). Methylthioadenosine (MA), uridine (U), adenosine (A), inosine (I), guanosine $(\mathrm{G})$, thymidine (T), deoxyuridine (DU), deoxyuridine (DA), deoxycytidine (DC) and deoxyguanosine (DG) were obtained from Aladdin Reagent Co., Ltd (Shanghai, China). HPLC grade acetonitrile (ACN) was used for the HPLC analysis. Ultrapure water was obtained from MilliQ gradient ultrapure water system (Millipore Inc., MA, USA). Anhydrous toluene was prepared by reflux with sodium. All the other chemicals were of analytical grade without further treatment.

\section{Preparation of the $\mathrm{SiO}_{2} @ H P G$ stationary phase}

The target stationary phase was prepared according to the procedure shown in Fig. 1. Prior to use, $\mathrm{SiO}_{2}$ was activated in a hydrochloric acid/ $\mathrm{H}_{2} \mathrm{O}(1 / 1, \mathrm{v} / \mathrm{v})$ solution with stirring for $24 \mathrm{~h}$. After the reaction, the $\mathrm{SiO}_{2}$ was washed thoroughly with deionized water to neutral $\mathrm{pH}$ and dried under a vacuum at $120^{\circ} \mathrm{C}$ for $24 \mathrm{~h}$.

The activated $\mathrm{SiO}_{2}(6.5 \mathrm{~g})$ was refluxed with CPTMS $(7.0 \mathrm{~mL})$ in anhydrous toluene $(100 \mathrm{~mL})$ with continuous stirring for $48 \mathrm{~h}$ under nitrogen. The product was centrifuged and washed successively with anhydrous toluene and anhydrous ethanol and dried under a vacuum at $40^{\circ} \mathrm{C}$. The obtained product was termed as $\mathrm{SiO}_{2} @ \mathrm{Cl}(6.7 \mathrm{~g}) . \mathrm{SiO}_{2} @ \mathrm{Cl}(6.2 \mathrm{~g})$ was weighed into DMF $(80 \mathrm{~mL}$ ) containing $9.0 \mathrm{~g}$ of Tris, and the suspension was heated at $80^{\circ} \mathrm{C}$ with stirring for $36 \mathrm{~h}$. The resulting $\mathrm{SiO}_{2} @$ Tris was collected by centrifugation and washed repeatedly with water until the aqueous supernatant was neutral. The $\mathrm{SiO}_{2} @$ Tris was then washed successively with methanol and acetone and dried under a vacuum overnight. $\mathrm{SiO}_{2} @$ Tris (3.2 g) was weighed into a round bottom flask equipped with a condenser and an oil bubbler. Dioxane $(60 \mathrm{~mL})$ containing PTB $(1.5 \mathrm{~g})$ was then added. The mixture was stirred and degassed with nitrogen for $30 \mathrm{~min}$ at $25^{\circ} \mathrm{C}$. The temperature was then raised to $95^{\circ} \mathrm{C}$. Glycidol $(12 \mathrm{~mL})$ was introduced dropwise under a nitrogen atmosphere and the reaction been continued for $24 \mathrm{~h}$. After polymerization, the mixture was cooled down to room temperature. The $\mathrm{SiO}_{2} @ \mathrm{HPG}$ was collected by centrifugation and washed repeatedly with water until the aqueous supernatant was neutral. The obtained material was dried under a vacuum at $40^{\circ} \mathrm{C}$ for $24 \mathrm{~h}$.

\section{Characterization}

FT-IR spectra were obtained on a Tensor-37 FT-IR spectrometer (Bruker Optics, Ettlingen, Germany) in the wavenumber range of $600-4000 \mathrm{~cm}^{-1}$ under ambient conditions. An attenuated total reflection (ATR) accessory was employed for all IR spectral acquisitions. Thermogravimetric analysis (TGA) was performed in a nitrogen atmosphere from room temperature to $1000^{\circ} \mathrm{C}$ with a heating rate of $10^{\circ} \mathrm{C} \mathrm{min}{ }^{-1}$ (Netzsch, Selb, Germany). 


\section{Column packing}

A slurry of $\mathrm{SiO}_{2} @ \mathrm{HPG}$ in methanol was prepared by ultrasonication for $2 \mathrm{~min}$ and packed into a stainless-steel column $(150 \times 4.6 \mathrm{~mm})$ under a constant pressure of $50 \mathrm{MPa}$ for $30 \mathrm{~min}$ with methanol as the pushing solvent. $\mathrm{SiO}_{2} @$ Tris packed column was prepared by the same procedure. Prior to chromatographic separation, the columns were conditioned with methanol at a flow rate of $1.0 \mathrm{~mL} \mathrm{~min}{ }^{-1}$ for $30 \mathrm{~min}$.

\section{Sample preparation and chromatographic conditions}

Stock solutions $\left(1 \mathrm{mg} \mathrm{mL}^{-1}\right)$ of the individual test analytes were prepared in pure water, pure $\mathrm{ACN}$ or a mixture of water and ACN. They were separately diluted with the corresponding mobile phase to obtain working solutions for HPLC injection. All of the tested polar analytes and their chemical structures are shown in Table 1 .

All of the chromatographic experiments were preformed on an Agilent 1200 series HPLC system, which consisted of an Agilent Quat pump, a degasser, a Rheodyne 7725i sample injection valve equipped with a $5-\mu \mathrm{L}$ loop, and a VWD UV detector. The flow rate was $1.0 \mathrm{~mL} \mathrm{~min}$. . Mobile phases were filtered through a $0.22-\mu \mathrm{m}$ membrane prior to use.

\section{Results and Discussion}

Characterization of the $\mathrm{SiO}_{2} @ H P G$ stationary phase

FT-IR was applied to characterize the feasibility of the preparation procedure. The spectrums of activated $\mathrm{SiO}_{2}, \mathrm{SiO}_{2} @$ $\mathrm{Cl}, \mathrm{SiO}_{2} @$ Tris and $\mathrm{SiO}_{2} @ \mathrm{HPG}$ are shown in Fig. 2(A). In trace b, the peaks at 2980 and $2892 \mathrm{~cm}^{-1}$ could be attributed to the $\mathrm{C}-\mathrm{H}$ stretching vibration, while the peak at $1404 \mathrm{~cm}^{-1}$ could be attributed to in-plane bending vibration of $\mathrm{C}-\mathrm{H}$. These results confirmed the graft of CPTMS onto the surface of $\mathrm{SiO}_{2}$. In trace c, the peaks at 3672,1388 and $649 \mathrm{~cm}^{-1}$ could be attributed to the stretching vibration, in-plane bending vibration and outof-plane bending vibration of $\mathrm{O}-\mathrm{H}$, respectively, while the peak at $1684 \mathrm{~cm}^{-1}$ could be attributed to an in-plane bending vibration of $\mathrm{N}-\mathrm{H}$, confirming the attachment of Tris through nucleophilic substitution reaction. Compared with trace c, the intensities of the peaks in trace $\mathrm{d}$ at $3672,2980,2892,1388$ and $649 \mathrm{~cm}^{-1}$ increased greatly while the intensity of the peak at $1684 \mathrm{~cm}^{-1}$ remained almost unchanged, which was due to the production of HPG on the surface of the stationary phase.

Thermogravimetric analysis (TGA) was carried out to further determine the graft of the HPG polymer on the surface of the silica particles. As shown in Fig. 2(B), the endothermic mass loss of the $\mathrm{SiO}_{2} @ \mathrm{HPG}$ stationary phase below $200^{\circ} \mathrm{C}$ was attributed to the loss of $\mathrm{H}_{2} \mathrm{O}$ molecules adsorbed by $\mathrm{SiO}_{2} @ \mathrm{HPG}$. From around $300^{\circ} \mathrm{C}$, the $\mathrm{SiO}_{2} @ \mathrm{HPG}$ demonstrated a rapid mass loss, indicating that the stationary phase had good stability at as high as about $250^{\circ} \mathrm{C}$. During $200-1000^{\circ} \mathrm{C}, 5.4$ and $5.7 \%$ weight loss was observed for the $\mathrm{SiO}_{2} @ \mathrm{Cl}$ and $\mathrm{SiO}_{2} @$ Tris, respectively, while $13.2 \%$ weight loss was found for $\mathrm{SiO}_{2} @$ HPG, indicating that the HPG polymer was successfully grafted onto the surface of silica particles.

\section{HILIC behavior evaluation}

Cytosine and uracil were selected as test solutes to evaluate the HILIC behavior of $\mathrm{SiO}_{2} @ \mathrm{HPG}$, and a comparison was made with $\mathrm{SiO}_{2} @$ Tris to assess the role of HPG. Uracil is less hydrophilic compared to cytosine, and the ratio $k_{\text {Cytosine }} / k_{\text {uracil }}$ has been suggested to be a function of the ACN content as a HILIC behavioral index. ${ }^{34}$ As shown in Fig. 3(A), the $k_{\text {Cytosine }}$ increased greatly, while $k_{\text {uracil }}$ increased slightly on $\mathrm{SiO}_{2} @ \mathrm{HPG}$ with
Table 1 Chemical structures of the tested analytes

\begin{tabular}{|c|c|c|}
\hline $\begin{array}{c}\text { Functional group } \\
\text { of analytes }\end{array}$ & Analyte & $\begin{array}{l}\text { Chemical } \\
\text { structure }\end{array}$ \\
\hline & Methylthioadenosine & \\
\hline & Uridine & \\
\hline & Adenosine & \\
\hline & Inosine & \\
\hline & Guanosine & \\
\hline & Thymine & \\
\hline or & Uracil & \\
\hline & Cytosine & \\
\hline & Guanine & \\
\hline & 2,5-Dimethylphenol & \\
\hline & Pyrocatechol & \\
\hline & 3-Nitrophenol & \\
\hline$\underbrace{S} \cdot{ }_{H} \cdot R$ & Sulfadimethoxine & \\
\hline & Sulfafurazole & \\
\hline & Sulfamethizole & \\
\hline & Sulfamethoxypyridazine & \\
\hline $\mathrm{HOH}_{2} \mathrm{C}-\mathrm{O}_{1}^{\mathrm{O}}$ & Thymidine & \\
\hline & Deoxyuridine & \\
\hline & Deoxyadenosine & \\
\hline & Deoxycytidine & \\
\hline & Deoxyguanosine & \\
\hline
\end{tabular}



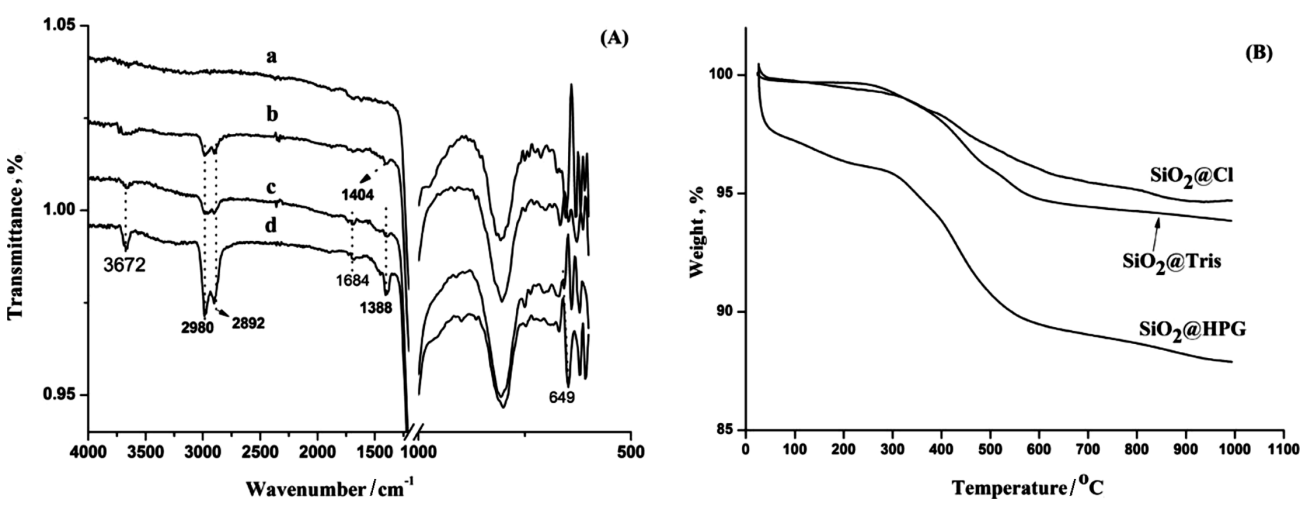

Fig. 2 (A) FT-IR spectra of activated $\mathrm{SiO}_{2}$ (a), $\mathrm{SiO}_{2} @ \mathrm{Cl}$ (b), $\mathrm{SiO}_{2} @$ Tris (c), and $\mathrm{SiO}_{2} @ \mathrm{HPG}$ (d). (B) TGA analysis of $\mathrm{SiO}_{2} @ \mathrm{Cl}, \mathrm{SiO}_{2} @$ Tris and $\mathrm{SiO}_{2} @ \mathrm{HPG}$.


Fig. 3 (A) Retention of uracil and cytosine on $\mathrm{SiO}_{2} @$ Tris and $\mathrm{SiO}_{2} @ \mathrm{HPG}$ with varying ACN content in mobile phase. Mobile phase: $\mathrm{ACN} / \mathrm{H}_{2} \mathrm{O}\left(50: 50\right.$, v/v) to $\mathrm{ACN} / \mathrm{H}_{2} \mathrm{O}(90: 10$, v/v), UV detection at 254 nm. (B) Separation of nucleosides on $\mathrm{SiO}_{2} @$ Tris and $\mathrm{SiO}_{2} @ \mathrm{HPG}$. Mobile phase: $\mathrm{ACN} / \mathrm{H}_{2} \mathrm{O}(85: 15$, v/v), UV detection at $260 \mathrm{~nm}$. Peaks: (1) methylthioadenosine (MA); (2) uridine (U); (3) adenosine (A); (4) inosine (I); (5) guanosine $(\mathrm{G})$; *, impurity in the sample.
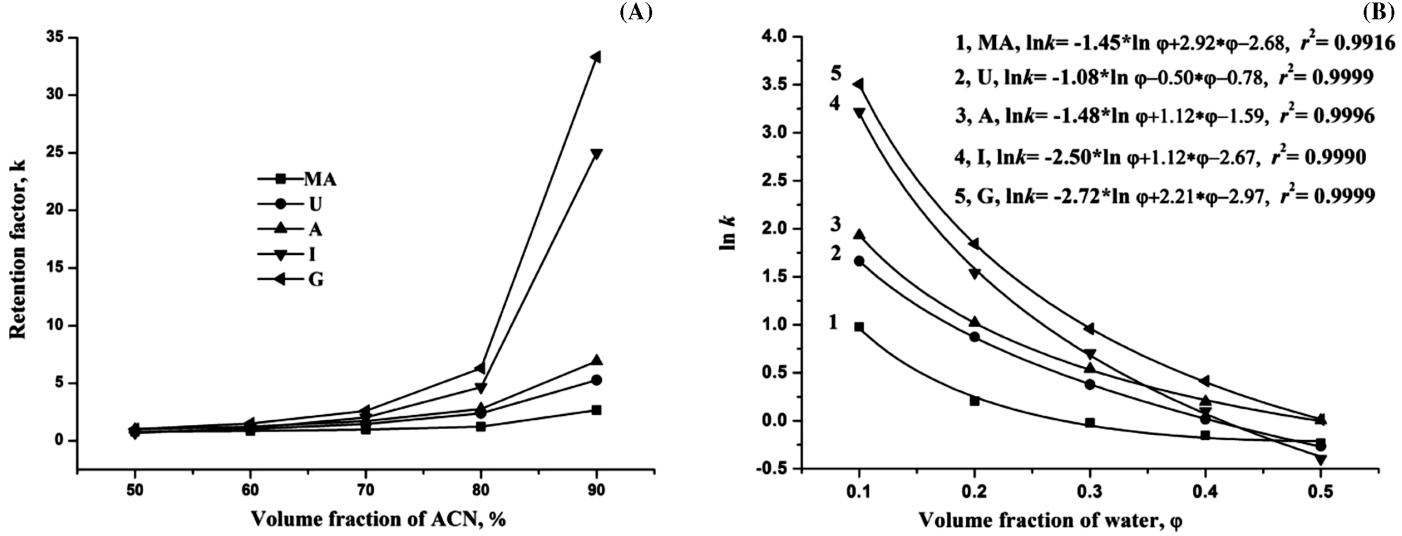

Fig. 4 (A) Plots of $\log k$ versus $\mathrm{ACN}$ fraction in the mobile phase for five nucleosides on $\mathrm{SiO}_{2} @ \mathrm{HPG}$. Mobile phase: $\mathrm{ACN} / \mathrm{H}_{2} \mathrm{O}\left(50: 50\right.$, v/v) to $\mathrm{ACN} / \mathrm{H}_{2} \mathrm{O}(90: 10$, v/v), UV detection at $254 \mathrm{~nm}$. (B) Plots of $\ln k$ versus volume fraction water for five nucleosides on $\mathrm{SiO}_{2} @ \mathrm{HPG}$. Mobile phase: $\mathrm{ACN} / \mathrm{H}_{2} \mathrm{O}$ (50:50, $\mathrm{v} / \mathrm{v})$ to $\mathrm{ACN} / \mathrm{H}_{2} \mathrm{O}(90: 10, \mathrm{v} / \mathrm{v})$, UV detection at $254 \mathrm{~nm}$.

increasing of ACN content. By comparison, $k_{\text {Cytosine }}$ and $k_{\text {uracil }}$ increased marginally on $\mathrm{SiO}_{2} @$ Tris with an increase of the $\mathrm{CAN}$ content. In addition, the ratio $k_{\text {cytosine }} / k_{\text {uracil }}$ for $\mathrm{SiO}_{2} @ \mathrm{HPG}$ increased much greater than that for $\mathrm{SiO}_{2} @$ Tris with the increase of the CAN content, indicating that $\mathrm{SiO}_{2} @ \mathrm{HPG}$ clearly exhibited the HILIC behavior. Figure 3(B) demonstrates that five nucleosides were well separated on $\mathrm{SiO}_{2} @ \mathrm{HPG}$. By contrast, those nucleosides could not be effectively separated on $\mathrm{SiO}_{2} @$ Tris under typical HILIC conditions.

The effect of the ACN concentration on the retention of five 


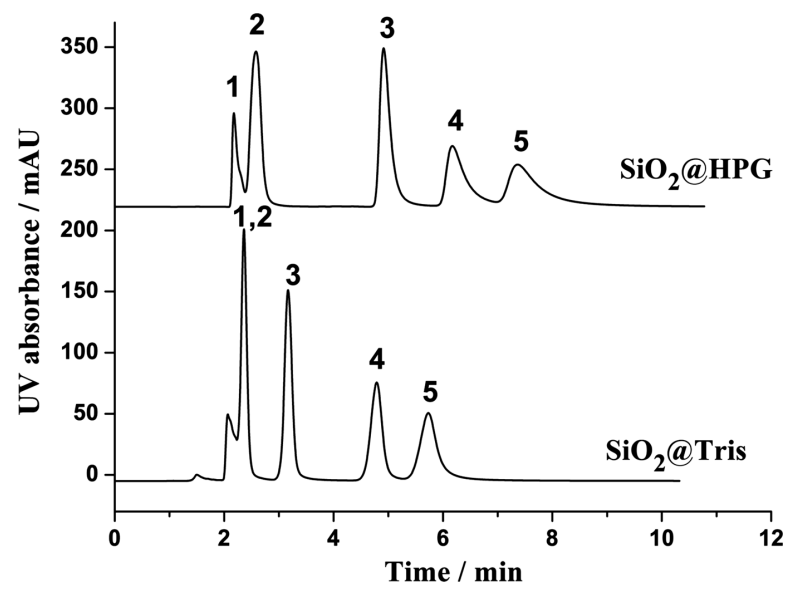

Fig. 5 Separation of nucleobases and nucleoside on $\mathrm{SiO}_{2} @$ Tris column and $\mathrm{SiO}_{2} @ \mathrm{HPG}$ column. Mobile phase: $\mathrm{ACN} / \mathrm{H}_{2} \mathrm{O}(80: 20$, $\mathrm{v} / \mathrm{v})$, UV detection at $254 \mathrm{~nm}$. Peaks: (1) thymine, (2) uracil, (3) cytosine, (4) guanine, (5) guanosine.

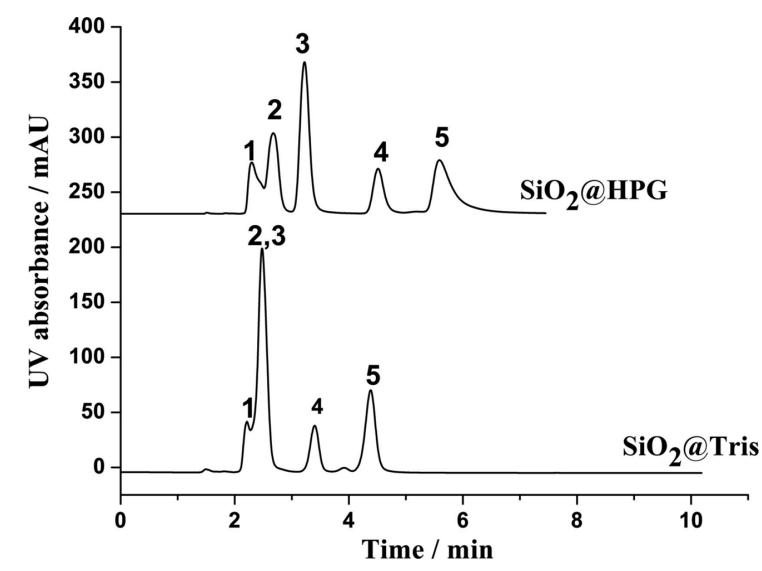

Fig.6 Separation of deoxynucleosides on a $\mathrm{SiO}_{2} @$ Tris column and $\mathrm{SiO}_{2} @ \mathrm{HPG}$ column. Mobile phase: $\mathrm{ACN} / \mathrm{H}_{2} \mathrm{O}(80: 20$, v/v), UV detection at $260 \mathrm{~nm}$. Peaks: (1) thymidine, (2) deoxyuridine, (3) deoxyadenosine, (4) deoxycytidine, (5) deoxyguanosine.

hydrophilic nucleosides was further investigated. As shown in Fig. 4(A), the logarithmic retention factors $(k)$ of the five nucleosides were plotted against the volume fraction of ACN in the mobile phase. Increasing retention factors were observed with increasing ACN content, demonstrating a typical HILIC retention characteristic.

Additionally, a quantitative HILIC model was studied based on Eq. (1), ${ }^{35}$ which was a dual mechanism model, taking both the partitioning and adsorption processes into consideration: ${ }^{36}$

$$
\ln k=a+b \ln \varphi+c \varphi,
$$

where $k$ is the retention factors $(a, b$, and $c)$ are constants, and $\varphi$ is the volume fraction of water in the mobile phase. As shown in Fig. 4(B), it demonstrated excellent fits (all of the regression coefficients $\left(r^{2}\right)$ were in the range of $\left.0.9916-0.9999\right)$ for the five nucleosides at five different mobile-phase compositions $(\varphi$ in the range of $0.10-0.50)$. The result indicated that the retention on the $\mathrm{SiO}_{2} @ \mathrm{HPG}$ stationary phase was based more on a mixed-mechanism rather than a simple partitioning or adsorption process.



Fig.7 Separation of phenols on a $\mathrm{SiO}_{2} @$ Tris column and a $\mathrm{SiO}_{2} @$ HPG column. Mobile phase: $\mathrm{ACN} / \mathrm{H}_{2} \mathrm{O}(90: 10$, v/v), UV detection at $254 \mathrm{~nm}$. Peaks: (1) 2,5-dimethylphenol, (2) pyrocatechol, (3) 3-nitrophenol.



Fig. 8 Separation of sulfanilamides on a $\mathrm{SiO}_{2} @$ Tris column and a $\mathrm{SiO}_{2} @ \mathrm{HPG}$ column. Mobile phase: $\mathrm{ACN} / \mathrm{H}_{2} \mathrm{O}$ containing $0.1 \%$ formic acid $(70: 30, \mathrm{v} / \mathrm{v})$, UV detection at $254 \mathrm{~nm}$. Peaks: (1) sulfadimethoxine, (2) sulfamethoxypyridazine, (3) sulfafurazole, (4) sulfamethizole.

Chromatographic performance comparison between $\mathrm{SiO}_{2} @$ Tris and $\mathrm{SiO}_{2} @ H P G$

To further evaluate the properties of the $\mathrm{SiO}_{2} @ \mathrm{HPG}$ stationary phase, the performance of the $\mathrm{SiO}_{2} @$ Tris and $\mathrm{SiO}_{2} @ \mathrm{HPG}$ was compared using necleobases, deoxynucleosides, phenols and sulfanilamides as probe analytes under the same chromatographic conditions.

Figure 5 depicts the separation of four nucleobases and a nucleoside on the $\mathrm{SiO}_{2} @$ Tris and $\mathrm{SiO}_{2} @ \mathrm{HPG}$ columns. The retention followed the polarity order, which was consistent with a typical HILIC retention mechanism, and the elution orders were the same on the two columns. The retention of the five analytes on the $\mathrm{SiO}_{2} @ \mathrm{HPG}$ was stronger than that on the $\mathrm{SiO}_{2} @$ Tris column, due to the improved hydrophilicity of $\mathrm{SiO}_{2} @ \mathrm{HPG}$. The five analytes were well separated on $\mathrm{SiO}_{2} @ \mathrm{HPG}$, while thymine and uracil coeluted on $\mathrm{SiO}_{2} @$ Tris. Figure 6 shows the separation of deoxynucleosides, and the separation performance was similar to that of necleobases with the coelution of DU and DA on $\mathrm{SiO}_{2} @$ Tris. These results demonstrated the improved separation ability due to the introduction of HPG. 
Chromatograms of three phenols are shown in Fig. 7; the three phenols could be well separated in the same eluting order on the two columns. On the $\mathrm{SiO}_{2} @$ Tris column, the peaks were broad and the theoretical plate numbers were 9454, 19115 and $6566(\mathrm{~N} / \mathrm{m})$ for DMP, PC and NP, respectively. In contrast, the $\mathrm{SiO}_{2} @ \mathrm{HPG}$ column showed favorable separation in a relatively shorter analysis time than the $\mathrm{SiO}_{2} @$ Tris column, and the theoretical plate numbers were improved to be 82455,45613 and $152463(\mathrm{~N} / \mathrm{m})$, respectively. In addition, the $\mathrm{SiO}_{2} @ \mathrm{HPG}$ column showed different selectivity from the $\mathrm{SiO}_{2} @$ Tris column (the selectivity factor $\alpha_{\mathrm{DMP} / \mathrm{PC}}=k_{\mathrm{DMP}} / k_{\mathrm{PC}}=1.61$ on the $\mathrm{SiO}_{2} @$ HPG column versus $\alpha_{\mathrm{DMP} / \mathrm{PC}}=1.31$ on the $\mathrm{SiO}_{2} @$ Tris column). The separation of four sulfanilamides on both columns is shown in Fig. 8 when the $\mathrm{SiO}_{2} @ \mathrm{HPG}$ column provided symmetrical peaks (asymmetry factor in the range of $1.01-1.16$ ) and good selectivity with efficiencies of $21378-50016(\mathrm{~N} / \mathrm{m})$. For comparison, the four sulfanilamides could not be separated and coeluted as one broad peak on the $\mathrm{SiO}_{2} @$ Tris column.

From the above comparisons, it was obvious that the modification of HPG on the surface of $\mathrm{SiO}_{2} @$ Tris could greatly improved the separation performance, providing improved peak shape and column efficiency as well as different selectivity towards various polar analytes.

\section{Conclusions}

In summary, a hyperbranched polyglycerol (HPG) functionalized silica stationary phase was prepared for HILIC analysis in a facial way. The abundant hydroxyl groups and hyperbranched structure of HPG offered good hydrophilicity, and the obtained stationary phase clearly demonstrated HILIC behavior. Nucleosides, nucleobases, phenols and sulfanilamides were well resolved on the $\mathrm{SiO}_{2} @ \mathrm{HPG}$ column, indicating great potential in biosamples separation. Furthermore, the alcohol functionality might lend itself to a variety of modifications, such as esterification and haloalkylation, to generate other types of stationary phases benefiting from the hyperbranched structure.

\section{Acknowledgements}

This work has been supported by the National Natural Science Foundation of China (grant No. 21405134, 21575123 and 21675139), the Jiangsu Science and Technology Project (BK20170471), the Talent Introduction Project of Yancheng Institute of Technology (XJ201509) and the Practical Innovation Program for Postgraduates of Specialty Degree in Universities of Jiangsu (SJLX16_0676). This work also sponsored by Jiangsu Qinglan Project and Jiangsu Industry University Foresight Joint Research Project (BY2016065-36).

\section{References}

1. A. J. Alpert, J. Chromatogr. A, 1990, 499, 177.

2. D. Garcia-Gomez, E. Rodriguez-Gonzalo, and R. CarabiasMartinez, Trends Anal. Chem., 2013, 47, 111.

3. H. Murakami, E. Otani, T. Iwata, Y. Esaka, T. Aoyama, M. Kawasaki, T. Tanaka, S. Minatoguchi, and B. Unot, Anal. Sci., 2015, 31, 1189.

4, L. Qiao, X. Shi, and G. Xu, Trends Anal. Chem., 2016, 81, 23.
5. P. Jandera and P. Janas, Anal. Chim. Acta, 2017, 967, 12.

6. F. E. Regnier and R. Noel, J. Chromatogr. Sci., 1976, 14, 316.

7. J. Wu, W. Bicker, and W. Lindner, J. Sep. Sci., 2008, 31, 1492.

8. A. J. Alpert, Anal. Chem., 2008, 80, 62.

9. D. S. Risley and M. A. Strege, Anal. Chem., 2000, 72, 1736.

10. Y. Guo and S. Gaiki, J. Chromatogr. A, 2011, 1218, 5920.

11. T. Ikegami, K. Tomomatsu, H. Takubo, K. Horiei, and N. Tanaka, J. Chromatogr. A, 2008, 1184, 474.

12. Q. Fu, Z. Guo, T. Liang, X. Zhang, Q. Xu, and X. Liang, Anal. Methods, 2010, 2, 217.

13. T. Yoshida, H. Hamada, H. Murakawa, H. Yoshimoto, T. Tobino, and K. Toda, Anal. Sci., 2012, 28, 179.

14. N. Takayama, L. W. Lim, and T. Takeuchi, Anal. Sci., 2017, 33, 619 .

15. H. Jiang, H. Yuan, Y. Qu, Y. Liang, B. Jiang, Q. Wu, N. Deng, Z. Liang, L. Zhang, and Y. Zhang, Talanta, 2016, 146, 225.

16. W. Shao, J. Liu, K. Yang, Y. Liang, Y. Weng, and S. Li, Talanta, 2016, 158, 361.

17. G. Huang, Z. Xiong, H. Qin, J. Zhu, Z. Sun, Y. Zhang, X. Peng, J. Ou, and H. Zou, Anal. Chim. Acta, 2014, 809, 61.

18. E. Wikberg, J. J. Verhage, C. Viklund, and K. Irgum, J. Sep. Sci., 2009, 32, 2008.

19. K. Wolski, M. Szuwarzynski, and S. Zapotoczny, Chem. Sci., 2015, 6, 1754.

20. P. Hemström, M. Szumski, and K. Irgum, Anal. Chem., 2006, 78, 7098.

21. W. Jiang, G. Fischer, Y. Girmay, and K. Irgum, $J$. Chromatogr. A, 2006, 1127, 82.

22. D. Yu, Z. Guo, A. Shen, J. Yan, X. Dong, G. Jin, Z. Long, L. Liang, and X. Liang, Talanta, 2016, 161, 860.

23. Y. Zheng, S. Li, Z. Weng, and C. Gao, Chem. Soc. Rev., 2015, 44, 4091.

24. D. Pranantyo, L. Xu, K. G. Neoh, E. T. Kang, and S. L. Teo, Ind. Eng. Chem. Res., 2016, 55, 1890.

25. M. A. Mata-Gómez, S. Yaman, J. A. Valencia-Gallegos, C. Tari, M. Rito-Palomares, and J. González-Valdez, J. Chromatogr. A, 2016, 1443, 191.

26. Y. Li, J. Yang, J. Jin, X. Sun, L. Wang, and J. Chen, J. Chromatogr. A, 2014, 1337, 133.

27. C. Kip and A. Tuncel, Chromatographia, 2017, 80, 565.

28. S. Lei, S. Yu, and C. Zhao, J. Chromatogr. Sci., 2001, 39, 280.

29. K. Sakai, T. C. Teng, A. Katada, T. Harada, K. Yoshida, K. Yamanaka, Y. Asami, M. Sakata, C. Hirayama, and M. Kunitake, Chem. Mater, 2003, 15, 4091.

30. M. Jáckowska, S. Bocian, and B. Buszewski, Analyst, 2012, 137, 4610.

31. S. Bocian, S. Studzinska, and B. Buszewski, Talanta, 2014, 127, 133.

32. Y. Peng, Y. Hou, F. Zhang, G. Shen, and B. Yang, Anal. Bioanal. Chem., 2016, 408, 3633.

33. M. Weinhart, I. Grunwald, M. Wyszogrodzka, L. Gaetjen, A. Hartwig, and R. Haag, Chem.—Asian J., 2010, 5, 1992.

34. N. P. Dinh, T. Jonsson, and K. Irgum, J. Chromatogr. A, 2011, 1218, 5880 .

35. Y. Hou, F. Zhang, X. Liang, B. Yang, X. Liu, and P. K. Dasgupta, Anal. Chem., 2016, 88, 4676.

36. G. Jin, Z. Guo, F. Zhang, X. Xue, Y. Jin, and X. Liang, Talanta, 2008, 76, 522. 